\title{
INJECTIVE MORPHISMS OF AFFINE VARIETIES
}

\author{
MING-CHANG KANG
}

(Communicated by Louis J. Ratliff, Jr.)

\begin{abstract}
In this note an elementary proof that every injective morphism from an affine variety into itself is necessarily surjective is given.
\end{abstract}

\section{INTRODUCTION}

Let $K$ be any algebraically closed field and $V$ an algebraic variety defined over $K$. It is known that any injective morphism from $V$ into itself is necessarily surjective $[7$, Proposition $(10.4 .11)$, p. $103 ; 1 ; 2 ; 6]$. Borel remarked that Shimura had a proof of this theorem by reduction modulo $p$ [6]. It seems that such a proof has not been published yet. The purpose of this note is to provide an elementary proof along this line when $V$ is an affine variety. What we shall prove is the following

Theorem. Let $K$ be any algebraically closed field, $V$ an affine variety defined over $K$, and $\varphi: V \rightarrow V$ a morphism from $V$ into itself. If $\varphi$ is injective, then it is surjective.

By [2, p. 3] the general case when $V$ is any algebraic variety follows from the above theorem. In fact, we shall present a proof for the general case in the appendix using Shimura's reduction theory, although we do not know whether this proof is what Shimura had in mind. For additional information when $V$ is an affine space see $[10 ; 5 ; 3,(2.1)$ Theorem].

\section{THE PROOF OF THE THEOREM}

The essence of our proof goes back to an idea of Shafarevich about $p$-group actions on affine spaces [4, Lemma; 8, Theorem 4.1].

Let $V$ be an affine variety in $\mathbf{A}^{n}$, the affine $n$-space. Denote the polynomial ring of $n$ variables over $K$ by $K\left[X_{1}, \ldots, X_{n}\right]$. Let $I$ be the defining ideal of $V$ and $g_{1}, g_{2}, \ldots, g_{s}$ a set of generators of $I$. Denote the coordinate ring of $V$ by

$$
R:=K\left[X_{1}, \ldots, X_{n}\right] / I=K\left[x_{1}, \ldots, x_{n}\right],
$$

Received by the editors October 30,1991 and, in revised form, January 8, 1992.

1991 Mathematics Subject Classification. Primary 14A10; Secondary 13B10.

Key words and phrases. Injective morphisms of affine varieties.

This work was done while the author was visiting Purdue University and was partially supported by the National Science Council of Republic of China. 
where $g(X)$ is regarded as an element in $K\left[X_{1}, \ldots, X_{n}\right]$, while $g(x)$ is regarded as an element in $R$. Points in $V$ or $\mathbf{A}^{n}$ will be denoted by $\left(a_{1}, \ldots, a_{n}\right)$, $\left(b_{1}, \ldots, b_{n}\right)$ or simply by $a, b$.

Let $\phi$ be a morphism from $V$ into $V$ given by

$$
\begin{aligned}
\phi: V & \rightarrow V \\
a & \mapsto\left(f_{1}(a), f_{2}(a), \ldots, f_{n}(a)\right)
\end{aligned}
$$

where $f_{1}(x), \ldots, f_{n}(x) \in R$. Let $V \times V$ be the product space of $V$ with itself, and consider the morphism

$$
\begin{aligned}
\Phi: V \times V & \rightarrow \mathbf{A}^{n} \\
(a, b) & \mapsto\left(f_{1}(a)-f_{1}(b), f_{2}(a)-f_{2}(b), \ldots, f_{n}(a)-f_{n}(b)\right) .
\end{aligned}
$$

Denote the diagonal of $V \times V$ by

$$
\Delta:=\left\{\left(a_{1}, \ldots, a_{n}, a_{1}, \ldots, a_{n}\right) \in V \times V: a_{i} \in K\right\} .
$$

It is clear that

$$
\begin{aligned}
& \phi \text { is injective } \\
& \quad \Leftrightarrow \Phi^{-1}(0, \ldots, 0)=\Delta . \\
& \quad \Leftrightarrow \text { there exists an integer } m \text { such that }
\end{aligned}
$$

$$
\left(x_{i}-y_{i}\right)^{m}=\sum_{j=1}^{n} h_{i j}(x, y)\left\{f_{j}(x)-f_{j}(y)\right\} \quad \text { for } 1 \leq i \leq n
$$

for some $h_{i j}(x, y) \in R \otimes_{K} R=K\left[x_{1}, \ldots, x_{n}, y_{1}, \ldots, y_{n}\right]$, where we identify $x_{i}$ and $y_{j}$ with $x_{i} \otimes 1$ and $1 \otimes y_{j}$ in $R \otimes_{K} R$.

Suppose that $\phi$ is not surjective and $c=\left(c_{1}, \ldots, c_{n}\right)$ is not in $\phi(V)$. Then the system of equations

$$
f_{1}(x)=c_{1}, \ldots, f_{n}(x)=c_{n}
$$

has no solution in $V$. Therefore, by Hilbert's Nullstellensatz, there exist $h_{1}(x)$, $\ldots, h_{n}(x) \in R$ so that

$$
\sum_{i=1}^{n} h_{i}(x)\left\{f_{i}(x)-c_{i}\right\}=1 .
$$

Collect all the coefficients of $g_{1}(X), \ldots, g_{s}(X), f_{1}(X), \ldots, f_{n}(X)$, $h_{i j}(X, Y), h_{i}(X)$, and all the $c_{1}, c_{2}, \ldots, c_{n}$. Call this set $\left\{d_{1}, d_{2}, \ldots, d_{t}\right\}$. Define a subring $S$ of $K$ by

$$
S:= \begin{cases}Z\left[d_{1}, d_{2}, \ldots, d_{t}\right] & \text { if } \operatorname{char} K=0, \\ Z_{p}\left[d_{1}, d_{2}, \ldots, d_{t}\right] & \text { if } \operatorname{char} K=p>0 .\end{cases}
$$

By Nagata's version of Noether's normalization lemma, there is a maximal ideal $M$ of $S$ so that $k:=S / M$ is a finite field [9]. by

Let $W$ be the affine $n$-space over $k$ and $W_{0}$ the affine variety in $W$ defined

$$
W_{0}=\left\{a \in W: \bar{g}_{1}(a)=\cdots=\bar{g}_{s}(a)=0\right\},
$$

where $\bar{g}$ is the image of $g$ when passing from $S$ onto $k$. 
Thus we obtain an injective morphism $\phi_{0}$ given by

$$
\begin{aligned}
\phi_{0}: W_{0} & \rightarrow W_{0} \\
a & \mapsto\left(\bar{f}_{1}(a), \ldots, \bar{f}_{n}(a)\right),
\end{aligned}
$$

which is not surjective because the image of $c=\left(c_{1}, \ldots, c_{n}\right)$ is in $W_{0}$ and both formulae (1) and (2) still hold when passing to $k$. Remember, both $W$ and $W_{0}$ are finite sets. Thus we get a one-to-one but not onto map from a nonempty finite set to itself-a contradiction.

\section{APPENDIX}

In this appendix we shall establish the theorem when the variety is any quasiprojective variety by applying Shimura's theory of reduction modulo $p$ of algebraic varieties [11;12, Appendix; 13, §9].

We recall some fundamental facts of Shimura's reduction theory. Let $K$ and $K^{\prime}$ be two fixed universal domains. We only deal with specializations defined on a subfield of $K$ taking values in $K^{\prime}$. Suppose that $k$ and $k^{\prime}$ are subfields of $K$ and $K^{\prime}$, respectively, $\lambda: k \rightarrow k^{\prime}$ is a specialization from $k$ onto $k^{\prime}$, and $V$ is a quasi-projective algebraic variety defined over $k$. Then $V$ has a unique specialization over $\lambda$, which we denote by $\bar{V}$. The notion of $\lambda$-simple varieties is introduced in [11, p. 163;13, p. 83]. For a $\lambda$-simple quasi-projective variety $V$, the specialization of $V$ over $\lambda$ preserves inclusion, sum, intersection-product, direct product, and projection [11, Theorems 17, 18, 19; 13, Proposition 1]. Moreover, if $V$ is irreducible and $x$ is a generic point over $k$, then, as a point set, $\bar{V}$ is equal to the set of all specializations of $x$ over $\lambda$ (into the universal domain $K^{\prime}$ ) [12, Lemma 3].

Now we may start to prove

Theorem A. Let $K$ be a universal domain, $V$ any quasi-projective variety over $K$, and $\varphi: V \rightarrow V$ a morphism from $V$ into itself. If $\varphi$ is injective, then it is surjective.

Proof. As in the proof of $\S 2$, define a subring $S$ of $K$ in a similar way so that both $V$ and $\varphi$ are defined over $k_{0}$ and $V$ has a $k_{0}$-rational point, where $k_{0}$ is the quotient field of $S$. By [12, Lemma 6] adjoin a finite number of nonzero elements of $k_{0}$ and their inverses to $S$ so as to assure $\lambda$-simplicity. By abuse of language we still denote by $S$ this enlarged finitely generated ring.

Again by Noether's normalization lemma, find a homomorphism $\lambda: S \rightarrow k_{0}^{\prime}$, where $k_{0}^{\prime}$ is some finite field. Extend $\lambda$ to a specialization of $k_{0}$; we still call it $\lambda$. Let $K^{\prime}$ be a fixed universal domain containing $k_{0}^{\prime}$. Then $\bar{V}$, the specialization of $V$ over $\lambda$, is defined over $k_{0}^{\prime}$ and is nonempty and $\lambda$-simple.

Let $\Gamma$ be the graph of $\varphi: V \rightarrow V$. Then $\bar{\Gamma}$, the specialization of $\Gamma$, is the graph of the endomorphism $\bar{\varphi}: \bar{V} \rightarrow \bar{V}$. Note that $\bar{\varphi}$ is injective again. For if $\xi$ is any point of $\bar{V}$ over $K^{\prime}$, choose a point $x$ of $V$ so that $\xi$ is a specialization of $x$ by [12, Lemma 3]. Then

$$
(\bar{V} \times\{\xi\}) \cdot \bar{\Gamma}=\overline{(V \times\{x\}) \cdot \Gamma}
$$

is either empty or consists of one point only. Assume that we have established the surjectivity of $\bar{\varphi}$. Then, again by (3), we find that $(V \times\{x\}) \cdot \Gamma$ is not empty; therefore, $\varphi$ is onto. 
To prove the surjectivity of $\bar{\varphi}$, it suffices to prove the surjectivity of $\left.\bar{\varphi}\right|_{\bar{V}\left(\overline{k_{0}^{\prime}}\right)}$, where $\bar{V}\left(\overline{k_{0}^{\prime}}\right)$ is the set of points on $\bar{V}$, whose coordinates are algebraic over $k_{0}^{\prime}$. Now let $k_{1}^{\prime}$ be any finite extension field of $k_{0}^{\prime}$ and $\bar{V}\left(k_{1}^{\prime}\right)$ the set of points on $\bar{V}$, whose coordinates are in $k_{1}^{\prime}$. Since $\bar{\varphi}: \bar{V}\left(k_{1}^{\prime}\right) \rightarrow \bar{V}\left(k_{1}^{\prime}\right)$ is an injective map of a nonempty finite set into itself, it is onto. Hence $\left.\varphi\right|_{\bar{V}\left(\overline{k_{0}^{\prime}}\right)}$ is surjective.

\section{REFERENCES}

1. J. Ax, The elementary theory of finite fields, Ann. of Math. (2) 88 (1968), 239-271.

2. __ Injective endomorphisms of varieties and schemes, Pacific J. Math. 31 (1967), 1-7.

3. H. Bass, E. H. Connell, and D. Wright, The Jacobian conjecture, Bull. Amer. Math. Soc. (N.S.) 7 (1982), 287-330.

4. A. Bialynicki-Birula, Remarks on the action of an algebraic torus on $K^{n}$, Bull. Acad. Polon. Sci. Sér. Sci. Tech. 14 (1966), 177-181.

5. A. Bialynicki-Birula and M. Rosenlicht, Injective morphisms of real algebraic varieties, Proc. Amer. Math. Soc. 13 (1962), 200-203.

6. A. Borel, Injective endomorphisms of algebraic varieties, Arch. Math. (Basel) 20 (1969), 531-537.

7. A. Grothendieck, Étude locale des schémas et des morphismes de schémas, EGA IV, Inst. Hautes Études Sci. Publ. Math. 28 (1966), 1-255.

8. M. Kang, Picard groups of some rings of invariants, J. Algebra 58 (1979), 455-461.

9. M. Nagata, Local rings, Interscience, New York, 1962.

10. D. J. Newman, One-one polynomial maps, Proc. Amer. Math Soc. 11 (1960), 867-870.

11. G. Shimura, Reduction of algebraic varieties with respect to a discrete valuation of the basic field, Amer. J. Math. 77 (1955), 134-176.

12. __ On the theory of automorphic functions, Ann. of Math. (2) 70 (1959), 101-144.

13. G. Shimura and Y. Taniyama, Complex multiplication of abelian varieties and its applications to number theory, Math. Soc. Japan, Tokyo, 1961.

Department of Mathematics, National Taiwan University, Taipei, Taiwan, Republic OF CHINA

E-mail address: kang@math.ntu.edu.tw 